\section{La dégradation}

\section{écologique au Rif}

marocain:

nécessités d'une

nouvelle

\section{approche}

\section{Taiqui'}

\section{RESUME:}

Au Nord du Maroc, les paysages montagneux du Rif sont soumis aux grands changements socio-économiques du XXe siècle. Ces changements sont à l'origine des contraintes démographiques, économiques et politiques affectant les structures naturelles et culturelles des paysages rifains traditionnels. Trois principaux aspects permettent d'interpréter la complexité des interactions entre les systèmes écologiques et socio-économiques au Rif: (1) l'accroissement des besoins alimentaires et énergétiques des populations locales qui est lié à la croissance démographique continue, (2) l'extension des cultures illicites du kif qui représentent la principale ressource économique de la région el (3) la nature de la politique nationale centrale, illustrée par les orientations et les instruments de gestion forestière. Les interactions entre les systèmes écologiques et ces différentes composantes socio-économiques et institutionnelles déterminent divers processus de dégradation du sol et perte de biodiversité.

L'insoutenabilité de la situation actuelle au Rif marocain nécessite de nouvelles stratégies de développement et de nouvelles approches de recherche scientifique. Dans l'objectif d'un développement soutenable, la connaissance des modèles d'organisation, de fonctionnement et d'évolution des paysages rifains est fondamentale pour fournir à la recherche écologique de nouvelles perspectives théoriques et appliquées.

\section{ABSTRACT:}

In the North of Morocco, the mountain landscapes of the Rif are submited to the strong socio-economic changes of the XXth century. These changes originate demographical, economical and political constraints that affect both natural and cultural structures of the traditional rifian landscapes. Three principal aspects permit to interpret the interactions complexity of ecological and socio-economical systems of the Rif: (1) the increase of nutritional and energetic needs of local populations who is connected to the continued demographic growth, (2) the illicit agriculture of the kif, who constitute the principal economic ressource of the region, and (3) the nature of the central national politic, illustrated by

\footnotetext{
' Département de Biologie, Faculté des Sciences, Université A. Essaâdi. B.P. 2121.93000 TETOUAN, MAROC.

Adresse actuelle: Departamento de Ecología, Universidad de Alicante. Ap. C. 99. 03080 ALICANTE, ESPAÑA.
}

the orientations and instruments of forestry management. The interactions between ecological systems and these different socio-economic and institutional components determines various processes of soil degradation and biodiversity loss.

The insustainable actual situation at the Rif mountains necesite new development strategies and new research approaches. In the objectif of a sustainable development, the knowledge of landscape patterns, function and changes is fundamental to provide new theoric and applicated perspectives for ecological research.

\section{INTRODUCTION:}

La dégradation écologique, au sens large du terme, est une propriété générale du système socio-économique actuel dont les impacts sont directement ou indirectement provoqués au niveau global (DI CASTRI \& HANSEN, 1992). Dans les pays en développement, les conséquences écologiques de cette globalisation sont particulières. Caractérisés jusqu'à une date récente par l'étendue de grands systèmes naturels ou semi-naturels, ces pays sont actuellement épuisés par la déforestation, la surexploitation et leurs conséquences écologiques liées aux transformations brutales et rapides des conditions socio-économiques. En Afrique méditerranéenne, le surpâturage et la forte densité humaine sont souvent évoqués dans l'interprétation de l'avancée du désert dans ces régions arides et semi-arides.

Dans le cas des montagnes du Rif, au Nord du Maroc (fig.1), la déforestation et le surpâturage liés à des grandes pertes des ressources édaphiques résument la situation actuelle des paysages sud-méditerranéens. Cependant, ces paysages montagneux, jeunes par leur géologie et leurs reliefs contrastés et attirants par leurs mosaïques de forêts, matorrals et champs cultivés, maintiennent encore plusieurs aspects de leurs structures traditionnelles. Malgré la pression démographique intense, le recours aux cultures illicites du kif (haschish ou Cannabis sativa var. indica) constitue une ressource économique très considérable; sans aller jusqu'à devenir une monoculture, les petites parcelles du kif assurent la vitalité des paysages rifains et renforcent leurs structures traditionnelles tout en transformant la région en une plate-forme internationale des réseaux de narco-trafiquants. Aujourd'hui, alors que les rapports nationaux et internationaux se multiplient condamnant ce type de culture et ses conséquences, les différentes solutions envisagées sont purement économiques et sont présentées dans le cadre général du même modèle de développement qui en est à l'origine.

Dans cet article, la complexité écologique au Rif est abordée en trois étapes: (1) l'examen des principaux facteurs naturels et socio-économiques intervenant au niveau du paysage, (2) la révision des conséquences écologiques de ces interventions selon les interprétations écologiques courrantes, et (3) la proposition d'un modèle hypothétique de recherche et d'aménagement écologique. 


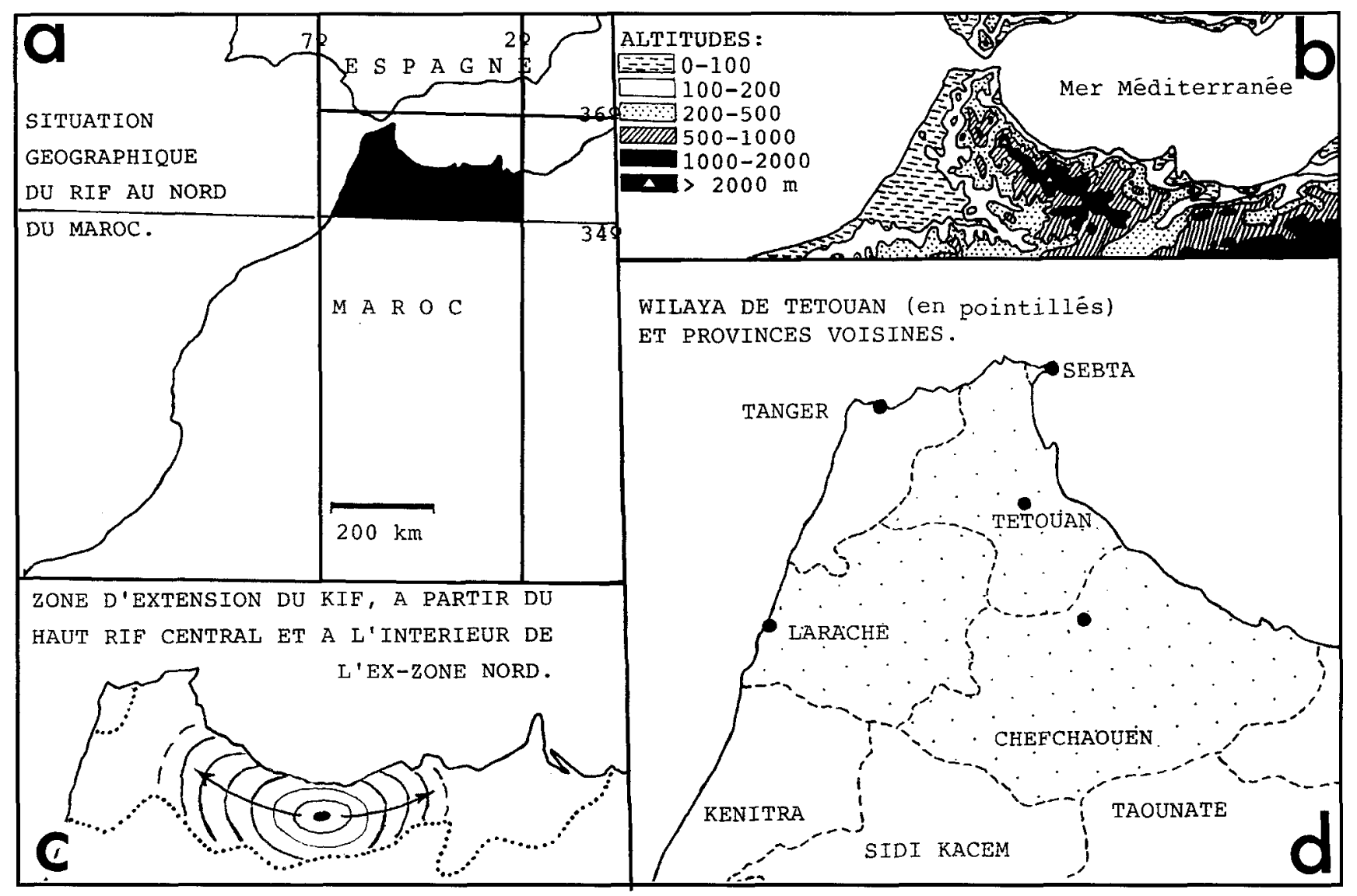

Fig. 1. Le Maroc, zone montagneuse d'extension du kif et provinces du Rif occidental.

A: Localisation du Rif au Nord du Maroc.

B: Représentation simplifiée des niveaux hypsométriques au Nord du Maroc.

C: Extension, à l'intérieur des limites de l'ex-zone Nord, des cultures du kif à partir des montagnes du Rif central.

D: Limites administratives des provinces du Rif occidental.

\section{LES DETERMINANTS ECOLOGIQUES ET SOCIO- ECONOMIQUES DES STRUCTURES ET DYNAMI- QUES DES PAYSAGES RIFAINS:}

Typiquement méditerranéen, le Rif, sur la rive Sud de la Mer d'Alboran, s'individualise par sa nature, son histoire et ses problèmes. Sa situation latitudinale, sa géologie, son orographie et son exposition aux influences maritimes humides représentent les principaux facteurs de son originalité naturelle (BENABID, 1982). De même, l'ancienneté de la population humaine et ses caractéristiques historiques et culturelles sont étroitement liées à l'évolution des paysages semi-naturels du Rif. Leur transformation actuelle est la résultante des conflits entre les dimensions culturelles et écologiques face aux phénomènes socio-économiques et institutionnels provoqués depuis le début du siècle. L'intégration de la dynamique écologique régionale dans son contexte global est particulièrement marquée par l'im- plication des forces du marché international des drogues (fig. 2).

\section{1\% Composantes naturelles et culturelles:}

Par rapport à l'ensemble des montagnes nord-africaines, la situation géographique privilégiée du Rif entre l'Atlantique et la Méditerranée, lui confèrent une originalité climatique dont la combinaison avec la diversité orographique et géologique régionale favorisent une grande richesse écologique (BENABID, 1983a). Dans la partie occidentale et centrale de ces montagnes, l'élévation altitudinale exposée à l'humidité et à la douceur des influences climatiques atlantiques et méditerranéennes favorisent le développement d'une végétation forestière luxuriante. Du thermoméditerranéen à l'oroméditerranéen, l'étagement de cette végétation est très affecté par l'hétérogéneité des expositions et des substrats géologiques. 


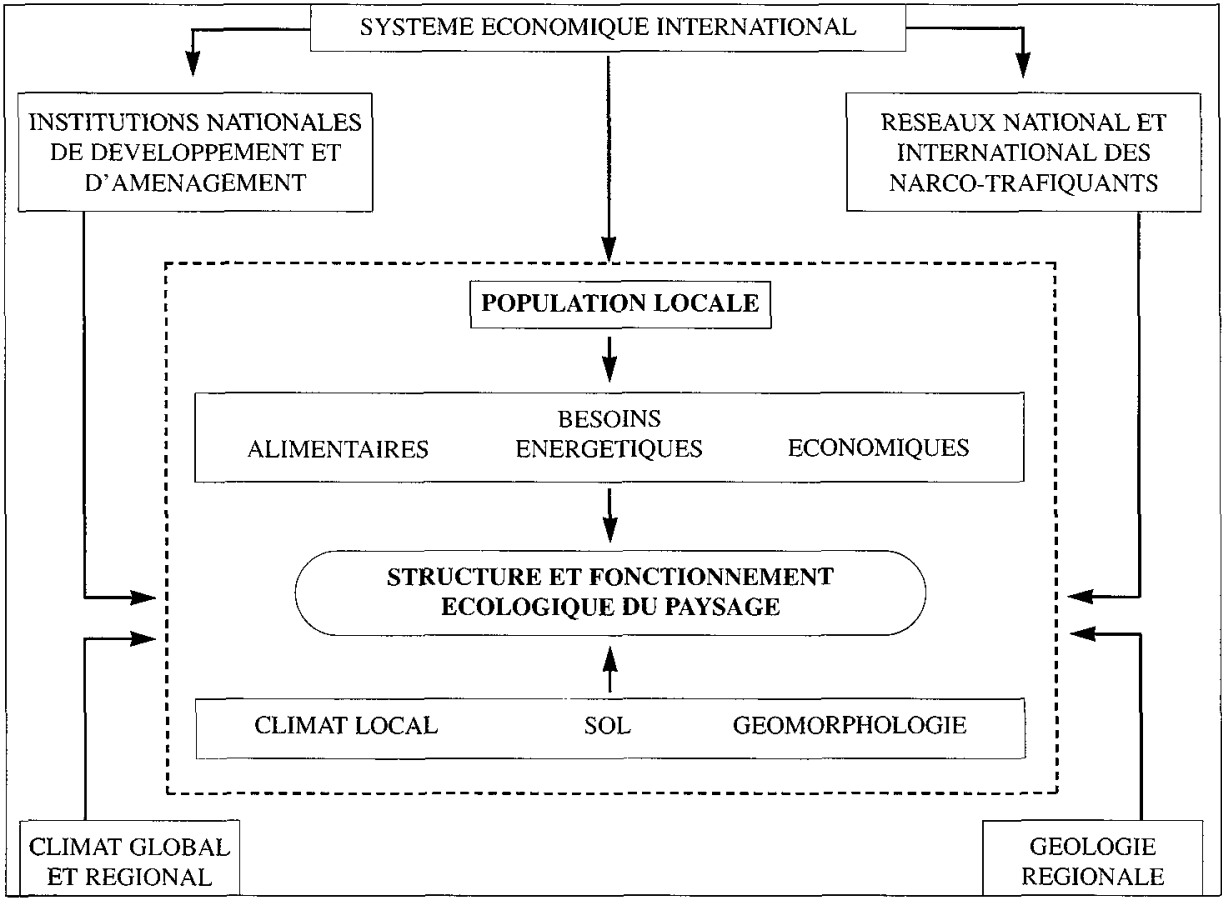

Fig. 2. Modèle schématique des principaux facteurs socio-économiques et écologiques intervenant dans la structuration et le fonctionnement des paysages rifains actuels.
En plus de ces facteurs naturels, l'ancienneté de la présence humaine constitue une composante fondamentale dans la structuration et le fonctionnement de ces paysages. Le conservationnisme caractéristique des montagnards, l'urbanisation historique qui remonte aux premières civilisations méditerranéennes, l'arabisation profonde et le rôle joué par la région dans le développement et l'épanouissement du sofisme marocain sont des constituants essentiels du paysage culturel rifain. A partir du XVI-XVIle siècles, suite à la stagnation économique du Maroc et son repli sur lui-même, la région a subit une régression générale vers un système économique de subsistance (TAIQUI \& MARTIN, 1997). D'un système de demande de productions commerciales naturelles et agraires variées, le Rif est passé à une économie autarcique dont la relation avec l'extension de la végétation naturelle est démontrée par les analyses polliniques (REILLE, 1977). Ces changements ont représenté au niveau du paysage la disparition de plusieurs centres urbains et l'organisation spatiale des agrosystèmes traditionnels sur de petites étendues limitées autour des hameaux et des petites villes persistantes. En montagne, ces agrosystèmes bordés de matorrals étaient, jusqu'à la veille du protectorat espagnol, presque entièrement noyés dans une matrice forestière hétérogène. Ces unités répétitives rappelant la structure typique du modèle de Von Thünen sont encore reconnaissables (EL GHARBAOUI, 1981; FAY, 1979; MAURER, 1968). Malgré les transformations socio-économiques de ce siècle et leurs répercussions certaines au niveau du paysage, la population locale semble, grâce à son passé historique, relativement mieux disposée à subir les conséquences du changement sans que cela entraîne une désintégration totale des agro-écosystèmes traditionnels.

En considérant l'exemple du seul paysage autour de Chefchaouen, ville-témoignage d'une histoire écologique riche et vivante, la haute diversité écologique, fortement concentrée dans l'espace, est représentée par une vallée majestueuse occupée par une subéraie étendue et dominée par les conifères et les forêts caducifoliées de montagne. Au niveau du massif calcaro-dolomitique de Talassemtane, QUEZEL et al. (1990), parlent, à propos du paysage endémique de la sapinière (Abies maroccana, Cedrus atlantica, Pinus nigra subsp. mauritanica, Acer granatensis, Quercus rotundifolia, Pinus pinaster subsp. maghrebiana), d'un véritable pôle de diversification des essences forestières en Afrique du Nord. Face à la dorsale calcaire, le massif gréseux de Bou-Hachem (Cedrus atlantica, Quercus pyrenaica, $Q$. canariensis, $Q$. rotundifolia, Pinus pinaster subsp. maghrebiana, Alnus glutinosa, Prunus lusitanicum, tourbières à Sphagnum auriculatum...) représente une valeur biologique exceptionnelle et un intérêt biogéographique irremplaçable (SAUVAGE, 1958).

La représentation verticale de ce paysage (fig. 3a) montre l'importance de l'hétérogéneité spatiale dominée par la couverture forestière. Elle indique l'étendue et l'étagement altitudinal de la végétation (du thermoméditerranéen au montagnard supérieur) liés aux variations climatiques naturelles (subhumide, humide et perhumide). La 

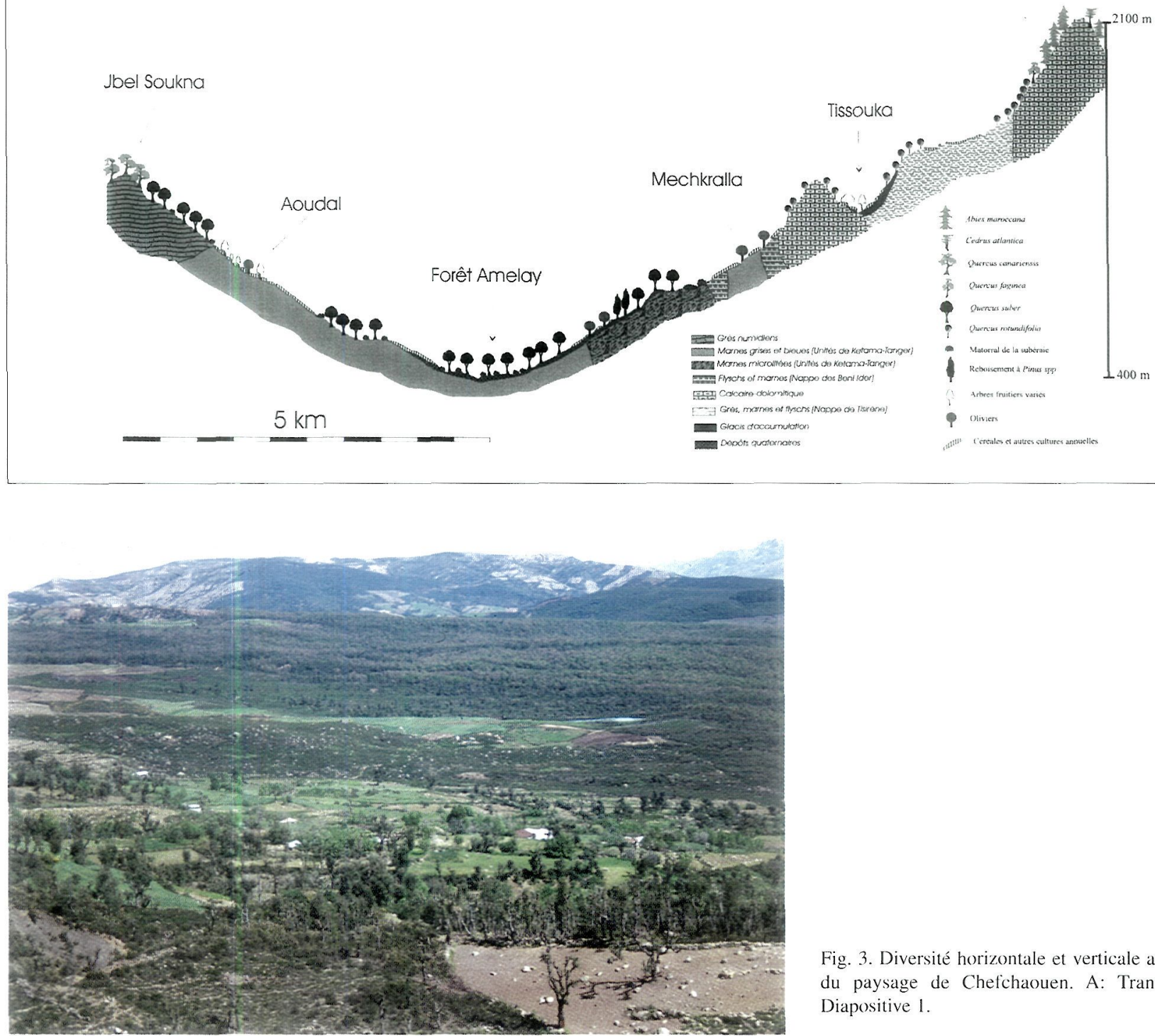

Fig. 3. Diversité horizontale et verticale au niveau du paysage de Chefchaouen. A: Transect, B: Diapositive 1.

variation lithologique contribue dans cette hétérogéneité en opposant la végétation calcifuge des terrains siliceux (Quercus suber et Q.canariensis) à la végétation calcicole des terrains calcaires (Q.rotundifolia, Q.faginea et Abies maroccana). Elle permet également de constater la position préférentielle des habitats humains et des occupations agricoles du sol au niveau du contact des roches dures avec les matériaux imperméables où jaillissent des sources d'eau permanentes. Ces habitats humains (dshar-s) dominent des cultures irriguées et fruitières auxquelles succèdent vers le bas des cultures extensives non irriguées.

Toutefois, l'observation directe de l'organisation hori- zontale des occupations du sol permet une meilleure lecture des interactions écologiques qui traversent le paysage (fig. 3b). Au milieu de la photographie, autour du noyau d'habitat humain traditionnel (Dshar Boubyen sur le versant Est du Jbel Bou-Hachem), la grande hétérogéneité suggère la présence d'une importante diversité naturelle au sein du "jnan" irrigué (cultures variées, haies, terrasses, arbres fruitiers, marabout constitué d'arbres naturels sacrés, etc.). Autour de ce système bocager, s'étendent des matorrals et des cultures extensives de céréales et légumineuses dont le contact avec la végétation naturelle indique une éventuelle expansion des parcelles cultivées. C'est ce 
que représente le premier plan de la photographie; la parcelle récemment défrichée contient encore quelques arbres de Chêne liège; à côté, le matorral à cistes est extrêmement appauvrit par le pâturage. La subéraie, au dernier plan, est rendue très hétérogène par les incendies, le parcours et l'exploitation du bois.

\section{2\% Particularités démographiques des montagnes du Rif occidental:}

Si du point de vue économique, l'accroissement démographique ne s'oppose pas à l'idée de croissance et de progrès continu, la vision globale de l'écologie affirme, au contraire, que cet accroissement est par définition incompatible avec un développement soutenable (EHRLICH, 1995). Dans les pays en développement, la poussée démographique et la pauvreté du milieu rural se traduisent généralement par une pression destructive des sols dont les conséquences sont largement représentées par l'exode rural. Selon SOMMA (1991), il s'agit plutôt d'un exode écologique dont les dimensions peuvent être énormes pendant certaines périodes critiques; c'est le cas par exemple en Afrique du Nord où, suite à la sécheresse des années 19811983, environ dix millions de personnes ont quitté les campagnes vers les portes des villes.

Au Maroc, la population totale est passée d'environ 4.5 millions d'habitants en 1900 à plus de 26 millions en 1994; le taux de population urbaine qui ne représentait pas plus de $1 \%$ au début du siècle, a rapidement atteint $51.4 \%$ en 1994 (REFASS, 1988; BENCHEIKH, 1995). Cette explosion démographique et l'exode rural qui l'accompagne concernent l'ensemble du territoire national. Cependant, la distribution humaine entre les milieux rural et urbain est très irrégulière d'une région à l'autre. A partir des données du recensement de 1994 (Direction de la Statistique, 1995), le taux de population rurale supérieur à $90 \%$ dans les Provinces de Chefchaouen et Taounate représente un cas extrême. Se maintenant si élevée et si opposée à la tendance nationale, la prédominance de la population rurale de ces deux provinces, occupant la majeure partie des montagnes du Rif central et occidental (fig. 1d), traduit des différences très prononcées relativement à l'ensemble du pays. Au niveau du Rif occidental, sans tenir compte de la population de la Province de Tanger $(83,8 \%$ de population urbaine), les différences entre Chefchaouen et les deux autres provinces de la Wilaya de Tétouan (Tétouan et Larache) sont très significatives (fig. 4). La Province de Chefchaouen se caractérise par la majorité écrasante de sa population rurale sensiblement égale à la somme des populations rurales de Tétouan et Larache (fig.4a). Le rythme de croissance démographique de cette population rurale de Chefchaouen est accéléré: de 25\% entre 1971 et 1982, il est passé à $42 \%$ entre 1982 et 1994 . Cette accélération de la croissance démographique dans les montagnes de Chefchaouen est illustrée par l'évolution du taux d'accroissement moyen annuel (fig.4b). Son augmentation est très considérable dans le milieu rural chefchaounais par rapport aux milieux rural et urbain dans le reste de la Wilaya.

En considérant l'interdépendance entre les besoins en

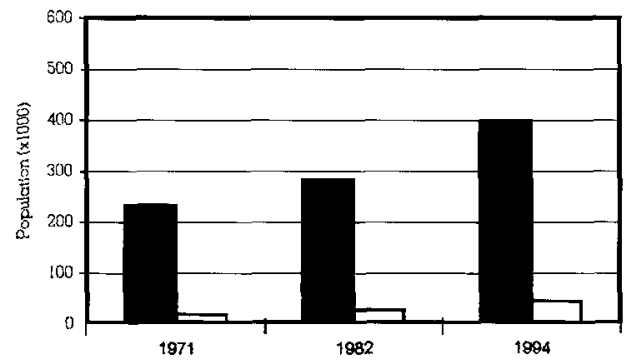

Chefchaouen

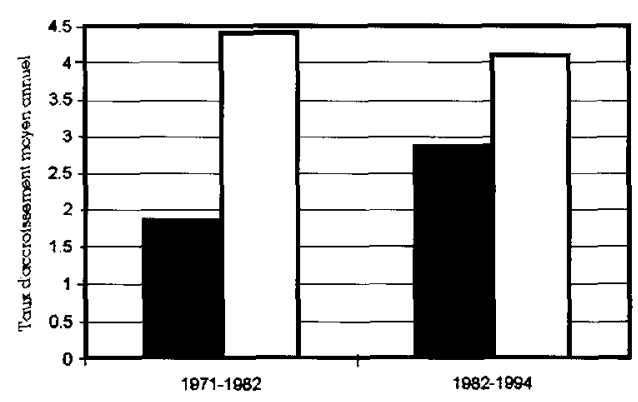

口Population urbaine

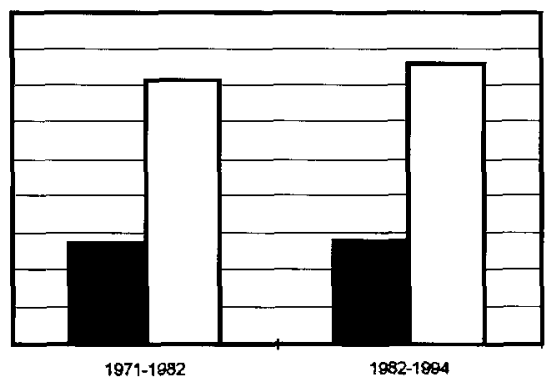

Fig. 4. -Particularités démographiques de la population rurale de la Province Chefchaouen en comparaison avec les deux autres provinces de la Wilaya de Tétouan. A: Croissance des populations urbaine et rurale, B: Taux d'accroissement moyen annuel de la population. 
ressources énergétiques et nutritionnelles (PIMENTEL et al., 1986), cet accroissement démographique de la population rurale, sans altération émigratoire notable, implique l'accroissement de l'exploitation du bois et l'extension des champs de cultures agricoles. L'ensemble des activités agrosylvopastorales des paysans doit fournir également d'autres revenus économiques nécessaires pour la subsistance en montagne tels que l'approvisionnement en moyens d'exploitation, matériaux et besoins domestiques. Il est donc tout à fait logique de considérer que ces fortes demandes nutritionnelles, énergétiques et économiques doivent se traduire de façon insoutenable au niveau social. Pour les besoins en combustible et les compléments fourragers, les femmes doivent se procurer, dans des conditions insupportables, du bois et des branchages dont les quantités sont de plus en plus insuffisantes (HAJJARABI, 1991). Leur vie est tellement occupée par la recherche du bois, qu'elles n'ont plus de temps à consacrer à l'éducation et à la nutrition des enfants. Ces derniers, très tôt intégrés dans les charges pénibles du travail rural, ne font que perpétuer pour les générations futures des conditions de plus en plus difficiles à

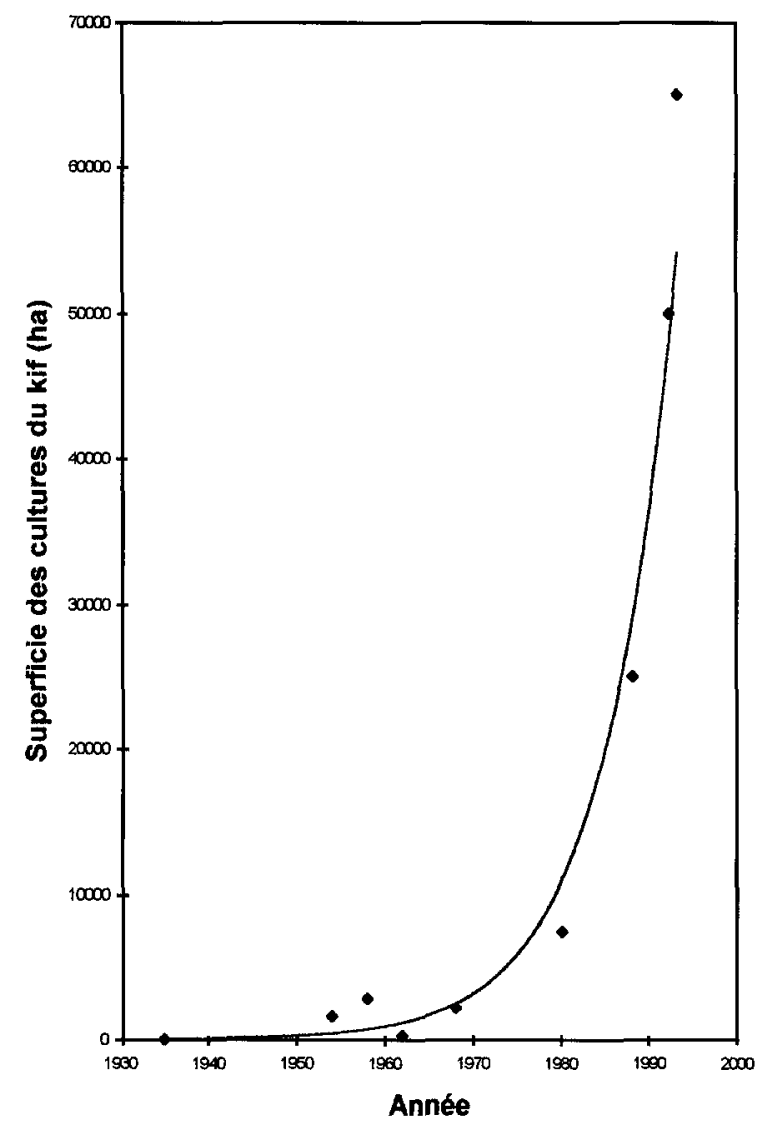

résoudre. En ville, la détérioration des conditions de vie et les problèmes urbains d'eau potable, décharges et épuration, pour ne citer que les besoins les plus élémentaires d'une vie urbaine saine, sont devenus insurmontables.

\section{3\% Les ressources économiques:}

Dans les montagnes rifaines, la grande densité humaine ne peut être expliquée que par la présence de ressources économiques capables de la supporter. Les paysans doivent subsister, et pour y parvenir, l'agriculture traditionnelle n'est plus suffisante. En fait, l'importante ressource économique des zones montagneuses du Rif central et occidental est incontestablement le haschish. Ses revenus d'environ $2000 \mathrm{~kg} / \mathrm{ha}$ (McNEILL, 1992) sont "20 à 30 fois supérieurs (...) à ceux d'une bonne récolte d'orge ou le double de la somme moyenne transférée dans l'année par un émigré en Europe" (MAURER, 1992b). Grâce à leur adaptation à la géographie et aux circonstances du marché (McNEILL, 1992), ces cultures illicites se sont rapidement étendues à partir du Rif central humide vers le Rif occidental et les zones semi-arides du littoral méditerranéen

\begin{tabular}{|c|c|c|}
\hline Année & Superficie (ha) & Source \\
\hline $1934-35$ & 21 & $\begin{array}{l}\text { RoDA JimENEZ (1941) } \\
\text { (In MAURER, 1968) }\end{array}$ \\
\hline 1954 & 1672 & $\begin{array}{l}\text { Enquête Officielle } \\
\text { (In MAURER, 1968) }\end{array}$ \\
\hline 1958 & 2909 & $\begin{array}{l}\text { Enquête Officielle } \\
\text { (In MAURER, 1968) }\end{array}$ \\
\hline $1961-62$ & 290 & $\begin{array}{c}\text { DERRO } \\
\text { (In MAURER, 1968) }\end{array}$ \\
\hline 1968 & $1500-3000$ & MAURER, 1968 \\
\hline 1980 & $5000-10000$ & O.G.D. (1994) \\
\hline 1988 & 25000 & MCNEILL (1992) \\
\hline 1992 & 50000 & $\begin{array}{c}\text { DisCOURS ROYAL } \\
\text { en } 1993\end{array}$ \\
\hline 1993 & 65000 & O.G.D. (1994) \\
\hline
\end{tabular}

Tableau I. Différentes estimations des superficies des cultures du kif durant les soixante dernières années.

Fig. 5. Expansion des superficies cultivées par le Cannabis au Rif. Les Points représentent les valeurs du Tableau I. Pour la courbe d'ajustemment exponentiel: $R^{2}=91 \%$ 
(fig.1c). A partir des estimations effectuées par différentes sources officielles et bibliographiques, l'ajustement exponentiel de l'expansion des cultures du kif en fonction du temps est hautement significatif (Fig. 5).

Par violation des lois officielles, les Jbala-s et les Rifains représentent peut être l'équivalent rural de l'explosion du secteur informel ou clandestin en ville, qui fait vivre des millions de personnes en démontrant "la défaillance du mode administratif de gestion et le prix trop élevé de la légalité" (SALAHDinE, 1991). Or, dans le cas du kif, il ne s'agit pas seulement d'une activité économique illicite. En effet, les dimensions des répercussions écologiques, culturelles, sociales, économiques et politiques du kif sont difficiles à cerner. Par sa grande rentabilité économique, il proportionne aux populations locales des revenus satisfaisants leur permettant de subsister tout en exposant la région aux dangers d'un système spécialisé et inflexible. Les dispositions gouvernementales prises dans les toutes dernières années et les pressions exercées par les pays européens pour trouver des solutions au problème du kif indiquent la gravité de la situation(ROYAUME DU MAROC, 1994).

Par ailleurs, plusieurs indices observés dans la région et rencontrés dans la littérature poussent à penser que les populations montagneuses investissent une partie importante des revenus du kif dans l'amélioration de l'agriculture et des conditions de vie en montagne. Une étude récente sur l'agriculture dans la Province de Chefchaouen indique, sans tenir compte des revenus des cultures du kif (difficiles à évaluer), que les différents types d'exploitations agricoles sont caractérisés par la diversité du cheptel et des cultures maraîchères, arboricoles, céréalières et légumineuses dont une grande partie est destinée au marché; la part des revenus hors exploitation ne dépasse pas $8 \%$ en moyenne (CHAKROUNI, 1994).

\section{4\% / La politique de gestion forestière:}

Datant du siècle dernier, longtemps avant l'apparition du concept de développement soutenable, le thème de la conservation des ressources environnementales n'est pas nouveau. Il n'est pas du tout étrange que, partout dans les pays en développement, les premières lois coloniales relatives à la législation forestière considéraient l'appropriation des domaines forestiers comme oeuvre "civilisationnelle" de protection du patrimoine naturel contre l'exploitation "anarchique" des indigènes. Dans ce cadre, la loi forestière marocaine, ordonnée autour du Dahir du 10-10-1917 relatif à la délimitation du domaine privé de l'Etat, a été "élaborée dans un souci de conservation et de développement, et renforcée par une fonction de contrôle qui assoie l'exercice du pouvoir de l'Etat" (Colloque National sur la Forêt, 1996). Selon PLIT (1983), à cette première phase d'élaboration du système législatif d'interdictions et d'ordonnances, succède la phase des travaux de Défense et Restauration des Sols par la dépossession des populations locales de leurs terres communales suivie de l'accélération des reboisements dont les travaux du projet Développement Economique et Rural du Rif Occidental(DERRO). Cette succession continue entre l'époque coloniale et post-coloniale a été soldée par un large échec (PLIT, 1983) dû à la nature des objectifs et aux contraintes imposées aux montagnards par l'administration au nom de l'intérêt national (MAURER, 1992a). C'est ce qui explique l'extension des défrichements permanents, les résultats médiocres des oliviers du DERRO ou leur refus par des villages et l'échec presque total de la tentative de "reconstitution du vignoble rifain" des années 1959-1964 (FAY, 1979).

Au Maroc, "à l'égard des projets et des idées techniques des administrateurs et des ingénieurs, la société rurale, dans son ensemble, manifeste une prudence expectative et une ferme résistance à des boulversements dont elle ne voit pas clairement l'issue et à la participation desquels elle ne préside pas" (PASCON, 1977). Selon FAY (1979), il faut redonner aux collectivités locales "une partie du pouvoir dont elles étaient dépossédées depuis la période coloniale";"toutes les connaissances empiriques qui existent dans la paysannerie doivent être revivifiées; il faut encourager les initiatives créatrices capables de rendre aux Jbala, sous des formes nouvelles, quelque chose de l'autonomie dont ils disposaient dans le passé". En réalité, ce genre d'appels à la participation des paysans, partout connu dans les pays en développement soumis aux recommandations des puissances économiques internationales (FUNNELL, 1994; STOCKING, 1995) a été précédé, au Maroc, par la naissance du Dahir du 20-9-1976 relatif à la participation de la population au développement de l'économie forestière. Malgré l'absence de textes d'application de ce Dahir, il s'agit bien d'une troisième phase des programmes d'aménagement sylvo-pastoral mais dont les résultats ne sont pas plus que des rapports et des recommandations (PLIT, 1983). Un de ces programmes sans effets durables est le projet Tanghaya au sud de Chefchaouen, où les apparences de réussite ont été rapidement démenties par l'incontournable invasion du kif (FAY, 1984).

A l'occasion du dernier Colloque National sur la Forêt (1996), le thème central était toujours le partenariat et l'appel à la participation des collectivités locales et des associations non gouvernementales. L'Administration forestière a rappelé également la carence dans l'aspect dissuasif et en matière de contrôle du matériel forestier tout en remettant en cause le droit d'usage qui, en raison de la pression démographique, n'est plus en équilibre avec les ressources forestières. Ses principaux objectifs demeurent ceux défi- 
nis par le Plan National de Reboisement (PNR) où l'on considère le Rif de façon particulière pour ses grandes potentialités forestières (BENABID, 1983b). Dans ce programme, le but stratégique de la politique nationale des reboisements est satisfaire les besoins nationaux par la création de 1970 jusqu'à l'an 2000 de 660000 hectares de peuplements artificiels dont les $2 / 3$ pour la production de bois d'oeuvre et d'industrie et le tiers restant pour la protection; le bois de feu n'est pas pris en considération (BENABID, 1991; HOLLOWAY, 1991).

\section{PROCESSUS DE TRANSFORMATION DES PAYSA- GES RIFAINS ET LEURS CONSEQUENCES ECO- LOGIQUES:}

Les contraintes socio-économiques et institutionnelles évoquées ci-dessus déterminent les différents proces- sus de dégradation écologique qui transforment les structures du paysage rifain et modifient son fonctionnement. La complexité des mosaïques actuelles du paysage et leur dynamisme sont largement contrôlés par le défrichement, les incendies, la surexploitation du bois et le surpâturage. Les principales conséquences écologiques sont représentées par des pertes considérables des ressources génétiques et édaphiques.

\section{1\% Principaux processus de dégradation au Rif:}

La coupe systématique pour l'exploitation industrielle ou commerciale protégée par les lois officielles est une forme directe, rapide et souvent irréversible de dégradation des écosystèmes forestiers rifains (fig. 6). La mise en culture par la coupe et le feu qui était autrefois une pratique courrante et localement planifiée dans le système tradi-
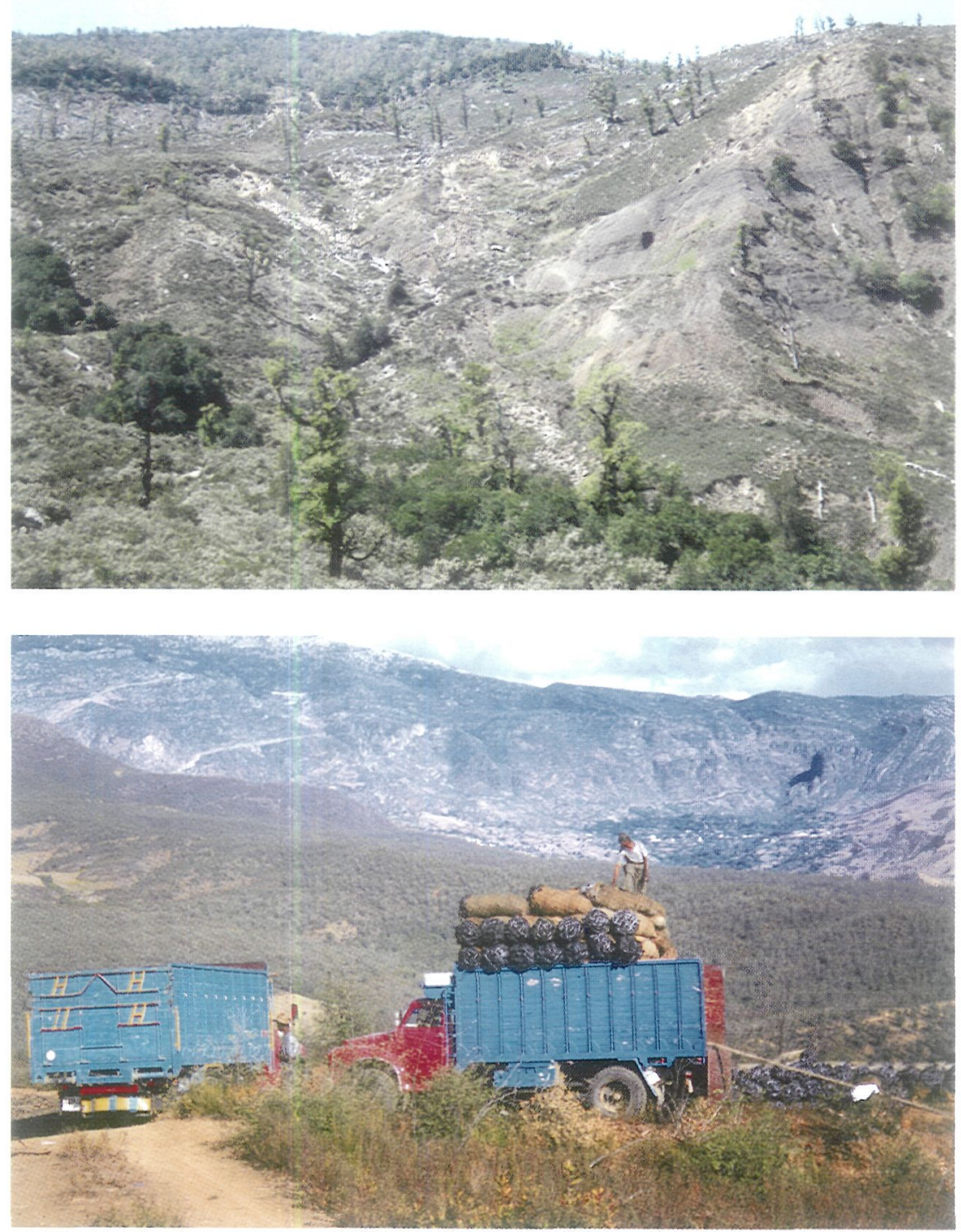

Fig. 6. Deux exemples d'explotation forestière protégés par les lois. A droite, la zénaie (Q. canariensis) de Bou-Hachem est dévastée par les services forestiers espagnols lors du Protectorat. A gauche, la subéraie (Q. suber) d'Amegri est confiée aux charbonniers après incendie, les systèmes racinaires des arbustes ne sont pas épargnés. Dans les deux cas, une même logique: répondre aux besoins socio-économiques insoutenables, et un même résultat: pertes irréversibles et incalculables du sol et de la biodiversité. Photographies prises en 1995 
tionnel des cultures itinérantes à longue rotation, est remplacée par des défrichements permanents. Les différents écosystèmes naturels sont également appauvris et déstructurés par le surpâturage essentiellement caprin et par la surexploitation de biomasse végétale pour les différents besoins énergétiques et commerciaux. En ce qui concerne les prélévements du bois de feu et de chauffage, les quantités estimées dépassent 6 tonnes par foyer et par an (MAURER, 1992b). De façon générale, on considère officiellement que $42 \%$ des forêts rifaines ont disparu lors des deux dernières décennies, ce qui correspond à 25 mille hectares par an. A ce rythme, bientôt il n'y aura plus de forêt rifaine (NACIRI, 1991). Dans le cas particulier des incendies, la région nord-ouest du Maroc perd environ 1185 ha/an, soit $43 \%$ de la superficie globale incendiée au niveau national (Colloque National sur la Forêt, 1996). En 1995, la superficie ravagée par le feu a atteint 6824 ha au niveau national dont 5800 ha concernent la seule région du Rif occidental.

\section{2\% L'érosion des sols:}

La première grande conséquence de ces différentes formes de déforestation est représentée par l'érosion des sols. Toutes les estimations effectuées au Maroc montrent que les valeurs les plus élevées de l'érosion sont enregistrés au Rif occidental et central. En fonction des bassins rifains et des méthodes d'estimation, ces valeurs varient entre 20 et $90 \mathrm{~T} / \mathrm{ha} / \mathrm{an}$ (Colloque National sur la Forêt, 1996; BROOKS et al., 1988). Si ces estimations sont discutables et ne donnent aucun renseignement ni sur la pédogenèse et la régénération édaphique ni sur les degrés d'irréversibilité de la dégradation du sol ni sur la part des activités humaines par rapport aux origines naturelles de l'érosion, il est par ailleurs certain que les quantités de sol qui envasent les barrages de la région et qui sont exportées vers la mer sont énormes. En ce qui concerne les risques et les conséquences de l'envasement des barrages rifains, elles sont catastrophiques (MARA \& MEFPFC, 1988). Quant aux sédiments exportés annuellement vers la mer, ils s'élèvent à 10,5 millions de tonnes pour les bassins méditerranéens et à environ 35,8 millions de tonnes pour les bassins de l'extrémité nord atlantique du Maroc (PROBST \& AMIOTTE SUCHET, 1992). Ces décharges augmentent de façon tragique lors des crues, comme c'est le cas pendant la catastrophe de Janvier-Février 1996.

Tout en considérant le caractère jeune et instable des reliefs, la fragilité du milieu naturel et sa forte susceptibilité à la pluviosité (BEAUDET et al., 1964), les différentes formes d'érosion sont largement amplifiées par les interventions humaines (EL GHARBAOUI, 1981). Cependant, peu de mesures défensives du sol, y compris les plantations forestières au niveau des versants, sont réellement protec- trices dans les conditions du Rif où les principaux phénomènes d'érosion représentés par le sapement des berges et les glissements de terrains nécessitent la stabilisation et la fixation biologique des réseaux hydriques (ROOSE, 1990). En plus de la protection du sol, les bénéfices des réseaux de végétation ripisylve sont nombreux et multiples. En permettant l'utilisation d'espèces autochtones et d'arbres fruitiers ou arbustes fourragers, de tels réseaux biologiques, peuvent rentabiliser le coût des aménagements et bénéficier de l'appui des populations riveraines tout en sauvegardant la biodiversité, la qualité des eaux et la stabilité écologique des paysages.

\section{$3 \%$ Les pertes de biodiversité:}

Les conséquences de la dégradation au niveau de la biodiversité sont innombrables. Depuis le début du siècle, la majorité des grandes espèces de différents groupes faunistiques ont disparu ou sont en voie d'extinction (ALAOUI, 1994). En plus du braconnage et différents modes de perturbation, les quelques espèces de Vertébrés qui sont actuellement protégées par la loi, sont surtout menacées par la réduction de leurs habitats naturels. L'état des Invertébrés est largement ignoré (DAKKI \& al., 1987).

En ce qui concerne les végétaux, parmi les grands écosystèmes forestiers du Rif, les oléastraies (Olea europa$e a$ var. silvestris) qui occupaient les sols fertiles à basses altitudes sont totalement éteintes et ne sont épargnées qu'au niveau des vestiges maraboutiques (BENABID, 1987). Les cocciféraies qui formaient de véritables forêts au Rif occidental ne sont plus visibles qu'au niveau des marabouts ou sous-forme de matorrals (BENABID, 1984). La situation des tétraclinaies et des pinèdes naturelles n'est pas très différente. Quant aux subéraies et chênaies vertes rifaines, elles restent assez étendues, mais fortement dégradées par différents modes d'exploitation et de dégradation. A haute altitude, la sapinière risque de disparaître dans un avenir très proche (BENABID, 1987, 1991). En plus de la déforestation et du surpâturage, les capacités de régénération naturelle de cette formation endémique sont fortement affaiblies par le désséchement des semis (MELHAOUI, 1990). QUEZEL (1991) considère que plus de la moitié de la superficie de la sapinière de Tazzaot a été perdue au cours des deux dernières décennies suite à des "incendies criminels". Si les sapinières et les cédraies, bénéficient d'un intérêt conservationniste particulier, les forêts caducifoliées de Q. canariensis qui ont été exploitées de façon régulière et systématique par les services forestiers espagnols semblent irrécupérables. La destruction de leurs sols humides et profonds empêche l'installation de toute végétation. Les quelques forêts caducifoliées qui persistent au Rif sont paradoxalement les plus étendues et les plus dégradées à l'échelle nationale (ZINE EL ABIDINE, 1988). 
De façon générale, les différents travaux concernant la biodiversité au Maroc ne cessent d'insister sur les nécessités de protection et de conservation. Toutefois, de nouvelles approches concernant la conservation écologique doivent être élaborées. Les appels à la création de parcs, réserves et collections vivantes d'espèces doivent élargir leur conception de l'écodiversité pour intégrer les dimensions biologiques, écologiques et culturelles des paysages seminaturels.

\section{POUR UNE NOUVELLE APPROCHE D'ETUDE ET D'AMENAGEMENT DU PAYSAGE:}

Le Rif résume les grands problèmes que le Maroc du XXIe siècle hérite d'une longue période de stagnation achevée par un siècle de boulversements. Les quelques aspects présentés dans cet article ne constituent en fait qu'une partie de cet héritage incompatible avec la survie et la persistance des systèmes socio-écologiques. Si la stabilité du Maroc et son avenir dépendent du développement de sa façade méditerranéenne (NACIRI, 1990), ce développement ne peut être conçu selon les modèles économiques classiques et insoutenables. La solution des problèmes et des conflits traversant les paysages rifains, dépendent de la société entière et nécessitent, entre autres, une haute considération des apports de la recherche scientifique. La technologie n'est pas la solution, mais peut être une partie de la solution (NAIMAN, 1992).

\section{$1 \%$ Développement soutenable et principes de précau- tion:}

Le développement soutenable, devenu largement utilisé par les chercheurs, les politiciens et le public informé, est un concept très problématique et difficile à définir et à prédire (COSTANZA \& PATTEN, 1995). Selon COCKLIN (1995), il s'agit d'un débat universel entre philosophies, idéologies et disciplines telles que l'environnementalisme et l'écologie, la science des ressources, l'économie néo-classique, les économies écologiques, l'économie politique et le postmodernisme.

Au niveau des études scientifiques, la littérature est dominée par des approches purement techniques au développement soutenable (GRAAF et al., 1996). Dans le cas des problèmes de l'érosion, par exemple, les résultats contradictoires de telles approches, dans les pays en développement, peuvent être attribués aux choix méthodologiques directement exposés aux influences de différents modèles idéologiques colonialistes (STOCKING, 1995). Certainement, l'élaboration d'une méthodologie d'approche scientifique à l'étude de la soutenabilité ne peut qu'être tributaire des divergences théoriques présentes. Etant donné la complexité des relations écologiques et socio-économiques au niveau du paysage, l'importance des connaissances scientifiques est fondamentale en tant qu'instrument de développement mais sans imposer les prescriptions scientifiques à la place des choix sociaux (LEE et al., 1992). Ces connaissances scientifiques doivent être basées sur l'ensemble des disciplines sociales, économiques et écologiques (PARK \& SEATON, 1996).

La complexité relative au concept de soutenabilité ou développement soutenable est beaucoup plus difficile à affronter par les services d'aménagement. Les réponses aux questions qui surgissent dans la réalité sont généralement risquées, incertaines, indéterminées ou le plus souvent ignorées. Tout en considérant les différences des institutions et des capacités technologiques et scientifiques entre les pays riches et les pays pauvres, ces degrés d'incertitude ne sont pas propres aux derniers; les systèmes naturels et leurs interactions avec les systèmes humains sont tellement complexes qu'ils échappent aux moyens courrants de modélisation mathématique et prédiction informatique de la soutenabilité (MOIR \& TODD MOWRER, 1995). Devant le dilemme "we have no answers, so what should we do?" (LUDWIG et al., 1993), le recours aux principes de précaution s'impose (DOVERS \& HANDMER, 1995). Les alternatives de restauration et de réhabilitation doivent avoir une perspective à long terme où les actions à entreprendre ne doivent pas dépendre des circonstances actuelles, mais de leurs retombées sur le futur. Cela suggère, dans le cas du kif par exemple, une extrême sensibilité vis-à-vis des conséquences certaines et éventuelles, immédiates et prolongées, que peuvent entrầner des mesures dictées par les circonstances actuelles.

\section{$2 \%$ Implications scientifiques:}

Considérés dans le cadre de la complexité des réalités écologiques, l'approche holistique et hiérarchique implique la présence, au niveau du paysage, de groupes d'écosystèmes interactuants, répétés de forme similaire et dont les propriétés sont supérieures à la somme des propriétés des écosystèmes constitutifs (FORMAN \& GODRON, 1986; FORMAN, 1994). Cette définition écologique du concept de paysage offre de nouvelles perspectives théoriques à une problématique spatiale largement débattue par les géographes et les agronomes (LEFEUVRE \& BARNAUD, 1988).

Quelles pourraient être ces systèmes répétitifs dans le cas des paysages rifains? Si plusieurs approches sont possibles en fonction des objectifs et des échelles de travail, dans le cas du Rif, et à partir des descriptions formulées plus haut, la considération des agro-écosystèmes traditionnels organisés autour des hameaux et des villages offre toutes les possibilités d'intégration des systèmes humains et naturels. A tous les niveaux scientifiques, la caractérisation de l'hétérogéneité de ces agroécosystèmes, leur évolu- 
tion dans le temps et l'étude des différents processus écologiques et leurs effects sur la dégradation de la biodiversité et du sol sont capables d'intégrer la recherche dans les problèmes d'aménagement et de développement du milieu rural rifain. A cette échelle spatiale, l'échelle temporelle correspondant aux séries de photographies aériennes disponibles qui couvrent environ un demi-siècle peuvent fournir une analyse complète de la stabilité ou instabilité des systèmes écologiques rifains. A la lumière d'une telle analyse, et en considérant différents aspects de l'hétérogéneité et sa relation avec la distribution des espèces, il est possible de prédire la sensibilité de ces systèmes par comparaison entre le capital biologique observé et la probabilité de le voir disparaître (GODRON, 1995).

Pour cela, le recours à l'élaboration de systèmes intégrés d'information géographique, capables d'incorporer les modèles de prédiction à des sources de données aussi variées que les images satellites, les photos aériennes, la documentation bibliographique et les recherches de terrain, est nécessaire. Cela permettra en plus d'un développement accéléré des connaissances relatives aux grandes lacunes sur les paysages rifains menacés, la quantification et la localisation précises des mutations spatiales ainsi qu'une véritable contribution scientifique aux alternatives de gestion, d'aménagement et de restauration.

\section{CONCLUSIONS:}

Des solutions idéales pour un développement idéal relèvent du domaine de l'utopie; les défis sont réels et imposent la recherche urgente d'alternatives réelles. Celles-ci doivent être globales; les remèdes partiels et aléatoires ne font qu'approfondir la crise. A partir des différents aspects examinés dans cet article, trois objectifs primordiaux sont concernés par un programme de développement économiquement et écologiquement soutenable au Rif: (1) freiner l'invasion rapide des paysages rifains par le kif, (2) améliorer les productions agro-sylvo-pastorales soutenables et (3) développer les connaissances scientifiques relatives aux interdépendances entre les systèmes naturels et humains.

En ce qui concerne le premier objectif, parler de "substitution" du kif est une formulation erronnée du problème car aucune activité économique légale ne peut lui résister dans les conditions concrètes du marché. A court terme, la situation est tellement compliquée qu'il n'est plus possible d'espérer plus que freiner la tendance actuelle. Compte tenu de son extension exponentielle et des incertitudes scientifiques actuelles, l'application des principes de précaution s'impose si l'on veut éviter des catastrophes sociales et écologiques imprévues.

En ce qui concerne l'amélioration de la production des systèmes agro-sylvo-pastoraux, elle doit être orientée vers l'objectif primordial visant à satisfaire les besoins alimentaires et énérgétiques de la population. Pour cela, l'augmentation de la productivité des systèmes traditionnels d'irrigation et d'arboriculture et le développement de l'agroforesterie dirigée vers la production du bois de feu, l'aménagement des pâturages et la protection des infrastructures écologiques constituent des objectifs de base tout comme le sont les projets d'installation des infrastructures physiques et sociales. Ce changement des conditions actuelles n'est nullement une affaire technocrate.

D'une part, les aspects socio-culturels doivent être hautement considérés; les premières préoccupations à ce propos doivent tourner autour de la scolarisation et la lutte contre l'analphabétisme, l'exploitation des femmes et la poussée démographique. D'autre part, le développement des connaissances scientifiques sur les structures et les dynamiques actuelles de fonctionnement des systèmes socio-écologiques du Rif doit être fortement intégré au processus de changement. En fait, les études descriptives font aboutir à des systèmes d'idées attractifs en théorie, coûteux et néfastes lors de leur confrontation avec la réalité. Les biologistes, écologues, agronomes, forestiers, pédologues et géographes, parmi les spécialistes les plus attachés aux thèmes de la conservation des ressources environnementales et l'amélioration des productions agricoles, doivent manifester leurs préoccupations par des recherches scientifiques concrètes. L'Université de Tétouan, principal établissement scientifique dans le Rif peut et doit jouer un rôle moteur à ce niveau. L'expérience du Groupe d'Ecologie de cette université dans le développement d'une activité scientifique collective, la coopération avec d'autres départements, services publics et établissements étrangers et l'acquisition d'une infrastructure de recherche constitue un bon exemple des possibilités d'action malgré l'atmosphère générale décourageante.

\section{Remerciements:}

L'auteur remercie E.SEVA-ROMÁN et C. MARTIN pour leurs remarques et corrections du manuscrit. Cet article a bénéficié de l'appui du Projet Med-Campus C-251 de la U.E..

\section{BIBLIOGRAPHIE :}

ALAOUI Y., 1996. Statut actuel de la faune sauvage en Afrique du Nord. Stratégie adoptée pour la protection des espèces rares et menacées d'extinction. $I_{n}$ V. PEIRO \& E. SEVA (Eds.), Conservación y explotación de la fauna en ecosistemas mediterráneos, Instituto de Cultura "Juan Gil-Albert", Alicante, 43-124.

BEAUDET G., MARTIN J. \& MAURER G., 1964. Remarques sur quelques facteurs de l'érosion des sols. Revue de Géographie du Maroc; 6: $65-72$.

BENABID A., 1982. Etude phytoécologique, biogéographique et dynamique des associations sylvatiques du Rif occidental. Problèmes 
posés par la reforestation et l'aménagement des peuplements forestiers actuels. Thèse Doctorat, Univ. St Jérome-Marseille, 199p.

BENABID A., 1983a. Etudes biogéographique et dynamique des peuplements forestiers du Rif (Maroc). Annales de la Recherche Forestière au Maroc, 1.23: 49-129.

BENABID A., 1983b. Problèmes posés par l'aménagement sylvopastoral et la réforestation dans le Rif centro-occidental (Maroc). Annales de la Recherche Forestiere cu Maroc, t.23: 385-423.

BENABID A., 1984. Etude phytoécologique des peuplements forestiers et préforestiers du Rif centro-occidental (Maroc). Trav. Inst. Sc., série Botanique, $\mathrm{n}^{\circ} 34:$ 1-64.

BENABID A., 1987. Les grands écosystèmes terrestres et leur préservation. G.E.M., Rabat, Vol. 4, 177-190.

BENABID A., 1991. La préservation de la forêt au Maroc. In M. REJDALI \& V.H. HEYWOOD Eds., Conservation des ressources végétales, Actes Editions, Rabat, p.97-104.

BENCHEIKH A., 1995. Population marocaine à travers les recensements. Revue Marocaine de Droit et d'Economie du Développement, 36 : 29-44.

BROOKS K.N., GREGERSEN H.M., BERGLUND E.R. \& TAYAA M., 1982. Economic evaluation of watershed projects - An overview methodology and application. Water Ressources Bulletin, 18 (2): 245-250.

CHAKROUNI A., 1994. Agriculture en zones de montagne et voies d'amélioration (région de Chefchaouen). Mém. Fin d'Etudes, I.A.V.Hassan II, Rabal, $211 \mathrm{p}$.

COCKLIN C., 1995. Agriculture, society and environment: discourses on sustainability. Int. J. Sustain. Dev. World Ecol., 2: 240-256.

COLLOQUE NATIONAL SUR LA FORET, 1996. Rapport des commissions préparatoires. Ifrane, Mars 1996.

COSTANZA R. \& PATTEN B.C., 1995. Defining and predicting sustainability. Ecological Economics, 15: 193-196.

DAKKI M., THEVENOT M., MEKOUAR M.A. \& KARMOUNI A., 1988. La protection de la faune. G.E.M., Rabat, vol. 10: 223-232.

DI CASTRI F. \& HANSEN A.J., 1992. The environment and development crises as determinants of landscape dynamics. In A.J. HANSEN \& F. DI CASTRI (Ed.), Landscape Boundaries. Consequences for Biotic Diversity and Ecological Flows, SpringerVerlag, New York, 3-18.

Direction de la Statistique, 1995. Population légale du Maroc. D'après le recensement général de la population et de l'habitat (Septembre 1994). Ministère Chargé de la Population, Rabat, 309p.

DOVERS S.R. \& HANDMER J.W., 1995. Ignorance, the precautionary principle, and sustainability. Ambio, 24(2): 92-97.

EHRILCH A.H., 1995. Implications of population pressure on agricultural and ecosystems. Advances in Botanical Research, 21: 79-104.

EL GHARBAOUI M., 1981. La terre et l'homme dans la Péninsule Tingitane. Etude sur l'homme et le milieu naturel dans le Rif occidental. Trav. Inst. Sc., Série Géologie te Géographie physique, $n^{\circ} 15,439 p$.

FAY G., 1979. L'évolution d'une paysannerie montagnarde: les Jbalas Sud-Rifains. Méditerranée, ${ }^{\circ} 1-2: 81-91$.

FAY G., 1984. Tanghaya. Un projet agro-sylvo-pastoral pour le Rif Occidental. Revue de Géographie du Maroc, n8, Nouvelle Série, 3-22.

FORMAN R.T.T, 1994. Land Mosaics. The ecology of landscapes and regions. Cambridge Univ. Press, Cambridge, 632p.

FORMAN R.T.T. \& GODRON M., 1986. Landscape Ecology. John Wiley, New York.

FUNNELL D.C., 1994. Intervention and indigenous management. The geography of small-scale irrigation development in Morocco and Swaziland. Land Use Policy, 11 (1):45-54.

GODRON M., 1995. Paisajes mediterráneos sometidos a perturbación. Consideraciones básicas. In A. PASTOR-LÓPEZ \& E. SEVAROMÁN (Ed.), Restauración de la cubierta vegetal en ecosistemas mediterráneos, Instituto de Cultura "Juan Gil-Albert", Alicante, 1320.

GRAAF H.J. de, MUSTERS C.J.M. \& KEUS W.J. ter, 1996. Sustainable development: looking for new strategies. Ecological Economics, 16: $205-216$.

HAJJARABI F, 1991. Sauver la forêt ou sauver les femmes: La corvée de bois chez les Ghmara. In Groupe Pluridisciplinaire d'Etude sur les Jbala éd., Jbala - Histoire et Société. Etude sur le Maroc du Nord-Ouest. Ed.CNRS-Paris, Wallada-Casablanca, 373-394.

HOLLOWAY C., 1991. La foresterie au Maghreb. In M. REJDALI \& V.H. HEYWOOD Eds., Conservation des ressources végétales, p.81-96, Actes Editions, Rabat.

LEE R.G., R. FLAMM R., TURNER M.G., BLEDSOE C., CHANDLER P., DEFERRARI C., GOTTFRIED R., NAIMAN R.J., SCHUMAKER N. \& WEAR D., 1992. Integrating sustainable development and environment vitality: a landscape ecology approach, In R.J. NAIMAN (Ed.), Watershed Management. Balancing Sustainability and Environmental Change, Springer-Verlag, New York, 499-521.

LEFEUVRE J.-C. \& BARNAUD G., 1988. Écologie du Paysage: mythe ou réalité. Bull.Ecol., t.19, 4, 493-522.

LUDWIG D., HILBORN R. \& WALTERS C., 1993. Uncertainty, ressource exploitation, and conservation: lessons from history. Science, 260: 17, 36 .

MARA (Ministère de l'Agriculture et de la Réforme Agraire) \& MEFPFC (Ministère de l'Equipement, de la Formation Professionnelle et de la Formation des Cadres), 1988. Séminaire national sur l'aménagement des bassins versants. Diagnosic de la situation actuelle. Rabat.

MAURER G., 1968. Les paysans du haut Rif central. Revue de Géographie du Maroc, nº 14: 3-70.

MAURER G., 1990. Facteurs physiques et aménagement dans la montagne rifaine. Revue Fac.Lettres Tétouan, 4e année, n4: 93-101.

MAURER G., 1992a. Agriculture in the Rif and Tell mountains of North Africa. Mountain Research and Development, 12 (4):337-347.

MAURER G., 1992b. Montagnes et montagnards au Maghreb (Maroc, Algérie, Tunisie). Evolution récente du milieu rural. Les Cahiers d'URBAMA, $\mathrm{n}^{\circ} 7: 36-61$.

McNEILL J.R., 1992. Kif in the Rif: a historical and ecological perspective on marijuana, markets, and manure in Northern Morocco. Mountain Research and Development, 12 (4):389-392.

MELHAOUI Y., 1990. Etude phytoécologique, productivité et classes de croissance du Sapin du Maroc (Abies maroccana Trab.). Problématique de la régénération naturelle des peuplentents de la sapinière marocaine. Thèse de doctorat, Univ. Aix-Marseille III, $200 \mathrm{p}$.

MOIR W.H. \& TODD MOWRER H., 1995. Unsustainability. Forest Ecology and Management, 75: 239-248.

NACIRI M., 1990. Le Maroc méditerranéen: l'envers du décor Groupement d'Etude et de Recherche sur la Méditerranée, Rencontre de Tétotuan, Rapport $\mathrm{n}^{\circ} 1,17 \mathrm{p}$.

NACIRI M., 1991. Rapport de synthèse. SOMADE, Rencontre: Eau et Environnement. Revue Marocaine de Droit et d'Economie du Développement, 26: 61-67.

NAIMAN R.J., 1992. New perspectives for watershed management: Balancing long-term sustainability with cumulative environmental change, In R.J. NAIMAN (Ed.), Watershed Management. Balancing Sustainability and Environmental Change, SpringerVerlag, New York, 3-11.

O.G.D. (Observatoire Géopolitique des Drogues), 1994. Etat des drogues, Drogue des Etats. Hachette-Pluriel, Série "Intervention", p.31-55.

PARK J. \& SEATON R.A.F., 1996. Integrative research and sustainable agriculture. Agricultural Systems, 50:81-100.

PASCON P., 1977. Le Haouz de Marrakech. Ed. Marocaines et Internationales, Tanger, 2 tomes.

PIMENTEL D., DAZHONG W., EIGENBRODE S., LANG H., EMER- 
SOM D. \& KARASIK M., 1986. Deforestation: interdependency of fuclwood and agriculture. OIKOS, 46: 404-412.

PLIT F., 1983. La dégradation de la végétation, l'érosion et la lutte pour protéger le milieu naturel en Algérie et au Maroc. Méditerranée, 3 : $79-88$.

PROBST J.L. \& AMIOTTE SUCHET P., 1992. Fluvial suspended sediment transport and mechanical erosion in the Maghreb (North Africa). Hydrol.Sc.J., 37(6): 621-637.

QUEZEL P., BARBERO M. \& LOISEL R., 1990. Les reboisements en région méditerranéenne. Incidences biologiques et économiques. Forêt méditerranéenne, 1.12 (2): 103-114.

QUEZEL P., 1991. Structures de végétation et flore en Afrique du Nord: leurs incidences sur les problêmes de conservation. In M. REJDA$\mathrm{Ll} \&$ V.H. HEYWOOD (Eds.), Conservation des resssources végétales. Proceedings. Actes Editions, Rabat, 19-33.

REFASS M., 1988. La population. G.E.M., Rabat, Vol.9: 8-36.

REILLE M., 1977. Contribution pollenanalytique à l'histoire holocène de la végétation des montagnes du Rif (Maroc Septentrional). Rech. franç. sur le Quaternaire, INQUA 1977, Suppl. Bull. A.F.E.Q., 1977-I(50): 53-76.

ROOSE E., 1990. Conservation des sols en zones méditerranéennes. La G.C.E.S, une nouvelle stratégie de lutte antiérosive, In U.I.M.P.
(Ed.), Soil erosion in Mediterranean environments, Valencia, 34p. ROYAUME DU MAROC, 1994. Livre Blanc. La politique générale du Maroc dans le domaine de lutte contre les drogues et pour le développement économique des provinces du Nord. Version arabe, $149 \mathrm{p}$.

SALAHDINE M., 1991. L'emploi invisible au Maghreb. Etudes sur l'économie parallèle. SMER, Rabat, $213 \mathrm{p}$.

SAUVAGE CH., 1958. Intérêt biogéographique du Bou-Hachem (Rif occidental). Société des Sciences Naturelles et Physiques du Maroc, t.38( ler trimestre): 17-26.

SOMMA M., 1991. Ecological flight: explaining the move from country to city in developing nations. Envirommental History Review, 15 (3): $1-26$.

STOCKING M., 1995. Soil erosion in developing countries: where geomorphology fears to tread! Catena, 25: 253-267.

TAIQUI L. \& MARTIN C.C., 1997. Elements historiques d'analyse écologique des paysages montagneux du Rif Occidental (Maroc). Mediterranea. Serie de estudios biológicos, 16:23-35.

ZINE EL ABIDINE A., 1988. Analyse de la diversité phyto-écologique des forêts du chêne zeen (Quercus faginea Lamk.) au Maroc. Bull. Inst. Sci., Rabat, 12:69-77. 